\title{
To study the Efficacy and safety of travoprost $0.004 \%$ BAK-free versus latanoprost $0.005 \%$ in patients with primary open-angle glaucoma or ocular hypertension.
}

\author{
Dr. Ramandeep Kaur ${ }^{1}$, Dr. Sachin Walia ${ }^{2}$, Dr. Harsimran Singh ${ }^{3}$, \\ Dr. Ishwar Singh ${ }^{4}$ \\ ${ }^{1}$ (Department Of Ophthalmology, Government Medical College Patiala, India) \\ ${ }^{2}$ (Department Of Ophthalmology, Government Medical College Patiala, India) \\ ${ }_{3}^{3}$ (Department Of Ophthalmology, Government Medical College Patiala, India) \\ ${ }^{4}$ (Department Of Ophthalmology, Government Medical College Patiala, India)
}

\begin{abstract}
:
Aim: To study the efficacy, safety, and tolerability of travoprost $0.004 \%$ benzalkonium chloride (BAK)-free versus latanoprost $0.005 \%$ in patients with primary open-angle glaucoma or ocular hypertension.

Settings and Design:

Prospective, randomized, comparative study in 30 patients of POAG/ocular hypertension with moderate glaucomatous damage were included.

Material and Methods:

The patients were randomly assigned to one of the two treatment groups, each having a sample size of 15 patients. All the patients were subjected to the detailed ocular examination at baseline and 12 weeks after starting the study treatment.

Results: No significant difference in mean intraocular pressure (IOP) was observed between latanoprost at baseline and travoprost BAK-free after 12 weeks nor were significant differences noted in mean ocular hyperemia, visual acuity, corneal fluorescein staining, or overall scores from the Ocular Surface Disease Index. Patients had a significantly shorter mean tear breakup time while on latanoprost compared with travoprost BAK-free.

Conclusion: The results of this study showed that both the groups had similar IOP control with better anterior segment signs in patients treated with travoprost BAK-free monotherapy.
\end{abstract}

Keywords: Intraocular pressure, Latanoprost, Travoprost, Primary open angle glaucoma, Ocular hypertension

\section{Introduction}

Prostaglandin (PG) analogues are the first-line IOP-lowering drugs because of their efficacy, systemic tolerability and once-daily regimen. Benzalkonium chloride (BAK) is one of the most potent preservatives and a component of most eye drops, because of its strong beneficial effect on microbacterial protection and solvent. ${ }^{1}$ On the other hand BAK-containing medications are associated with increased ocular surface damage, and decreased tolerability compared with BAK-free preparations as suggested by many studies ${ }^{2-4}$.Latanoprost $0.005 \%$ contains $0.02 \% \mathrm{BAK}$, one of the highest BAK concentrations found in ophthalmic preparations. Instead of using BAK as a preservative, travoprost $0.004 \%$ BAK-free contains the alternative preservative system SofZia, containing borate, zinc, and sorbitol ${ }^{3}$. BAK-free PG solutions showed similar IOP-lowering effect to BAK-preserved solutions and more favourable effects on discomfort, dry eye or foreign body sensation, which are common symptoms of OSD in many studies ${ }^{5.6}$ Discomfort, dry eye or foreign body sensation, which are common symptoms of OSD, may lead to poor patient adherence to glaucoma treatment. Thus, treating glaucoma patients with eye drops with minimal or no BAK should not only minimize the occurrence of OSD but also increase the adherence to treatment. Thus the goal of the current study was to examine the efficacy, safety, and tolerability of travoprost $0.004 \%$ BAK-free compared with latanoprost $0.005 \%$ in patients with primary open angle glaucoma or ocular hypertension.

\section{Materials and Methods}

In this prospective, randomized, comparative study, 30 patients of POAG/Ocular Hypertension with moderate glaucomatous damage attending the Outpatient Department of Ophthalmology, at our institute were included. The patients were randomly assigned to one of the two treatment groups, each having a sample size of 15 patients.

Group 1): consisted of 15 patients of primary open angle glaucoma/ocular hypertension .This group instilled 1 drop of latanoprost $0.005 \%$ at 8 p.m. (once daily ) for 12 weeks. 
Group 2): consisted of 50 patients of primary open angle glaucoma/ocular hypertension. This group instilled 1 drop of travoprost $0.004 \%$ benzalkonium chloride (BAK)-free at 8p.m. for 12 weeks. All the patients were subjected to the following examination and tests at baseline, 4 weeks, 8 weeks and 12 weeks after starting the study treatment :

- Best corrected visual acuity using Snellen's chart.

- Detailed ocular examination including eye lashes, lid and adnexa was done using diffuse light.

- Conjuctival hyperaemia

- Tear film break up time for dry eye

- Corneal fluorescein staining,

- Goldmann applanation tonometry was used to measure intraocular pressure.

- Angle of anterior chamber was assessed by doing gonioscopy with Goldmann goniolens to exclude the cases of angle closure glaucoma.

- Cornea, iris, lens and pupil were examined.

- At the baseline visit, patients were asked to complete the validated Ocular Surface Disease Index (OSDI) questionnaire. OSDI scores were classified as normal (0-12), mild ocular surface disease symptoms (1322), moderate ocular surface disease symptoms (23-32), and severe ocular surface disease symptoms (33$100)^{7}$.

Whole data was recorded and analyzed using statistical tests:

\section{Results}

No significant difference in mean IOP was observed between latanoprost monotherapy and travoprost BAK free monotherapy after 12 weeks ( $p$ value 0.793 ) nor were significant differences noted in mean ocular hyperemia ( $p$ value $\square 0.060$ ). Patients had a significantly shorter mean tear breakup time while on latanoprost monotherapy compared with travoprost BAK-free monotherapy ( $p$ value 0.011). No significant changes were there in visual acuity, corneal staining, or slit-lamp biomicroscopy. Mean OSDI scores remained normal throughout the study.

Age Range: $74.57 \pm 9.51$

Table 1: Gender

\begin{tabular}{|l|l|l|}
\hline Gender & No. of Patients & Percentage \\
\hline Female & 17 & $56.67 \%$ \\
\hline Male & 13 & $43.33 \%$ \\
\hline Total & 30 & $100 \%$ \\
\hline
\end{tabular}

Table 2: Mean efficacy and safety measure

\begin{tabular}{|l|l|l|l|l|l|}
\hline Measure & $\begin{array}{l}\text { Latanoprost } \\
(\text { Mean } \pm \text { SD) }\end{array}$ & $\begin{array}{l}\text { Travoprost BAK-free } \\
(\text { Mean } \pm \text { SD) }\end{array}$ & t-test & $p$ value & $\begin{array}{l}\text { Sign } \\
.\end{array}$ \\
\hline Intraocular Pressure (mmHg) & $17.93 \pm 2.73$ & $18.13 \pm 3.12$ & 0.264 & 0.793 & NS \\
\hline Tear film breakup time (sec) & $9.53 \pm 3.67$ & $11.97 \pm 3.53$ & 2.624 & 0.011 & S \\
\hline $\begin{array}{l}\text { Ocular Hyperemia (scale of 0- } \\
\text { 3) }\end{array}$ & $0.75 \pm 0.81$ & $0.41 \pm 0.55$ & 1.918 & 0.060 & NS \\
\hline
\end{tabular}

\section{Discussion}

Incidence of ocular surface disease (OSD) in glaucoma patients is higher than the general population because they are usually treated with a variety of ocular hypotensive drugs with various preservatives throughout their lives. The introduction of BAK containing eye drops was associated with an approximately twofold greater likelihood of developing OSD for each additional medication ${ }^{8}$.So various kinds of preservatives have been alternatively used instead of BAK in PG analogues. Travoprost introduced three types of ophthalmic solution with SofZia and polyquad in addition to its original solution with BAK. Regarding to SofZia-preserved travoprost, 8 weeks or 3 months following the transition from BAK preserved PG to SofZia-preserved travoprost,elements of OSD, tear break-up time (TBUT) and the ocular surface disease index (OSDI) - scores that quantify dry eye symptoms improved ${ }^{4,6}$ However, these previous study designs were one-way switching and not randomized comparative studies. Lewis et $\mathrm{al}^{5}$ directly compared BAK-preserved travoprost and SofZiapreserved travoprost by a double-masked, randomized, parallel group, multicentre design. In this study, there was no difference between two groups in IOP-lowering effect and the frequency of adverse event. Thus, we conducted a study to compare benzalkonium chloride (BAK)-free travoprost $0.004 \%$ and latanoprost $0.005 \%$ in patients with primary open-angle glaucoma or ocular hypertension. In the current study, patients had a mean increase of almost two seconds in tear breakup time after 12 weeks of travoprost BAK-free monotherapy. 
Studies conducted by Michael $\mathrm{J}$ Miyashiro et $\mathrm{al}^{9}$ and the Horsley study, which compared latanoprost with travoprost BAK-free also reported a significant increase in tear breakup time after transition to travoprost BAKfree supporting the results of our study. Patients in the current study were found to have no significant changes in IOP after12 weeks in both the groups. So the removal of BAK does not affect IOP control. Ocular hyperemia was less with travoprost BAK-free, but was statistically insignificant. No significant changes were observed in other parameters like visual acuity or corneal staining.

\section{Conclusion}

This study demonstrates that the patients of primary open-angle glaucoma or ocular hypertension can be given Travoprost $0.004 \%$ BAK-free monotherapy in place of latanoprost $0.005 \%$ monotherapy with similar IOP control. Also this drug had not much safety issues as compared to other prostaglandins and was tolerable by the patients.

\section{Bibliography}

[1]. Kaur IP, Lal S, Rana C, Kakkar S \& Singh H(2009): Ocular preservatives: associated risks and newer options. Cutan Ocul Toxicol 28: 93-103.

[2]. Kahook MY, Noecker RJ. Comparison of corneal and conjunctival changes after dosing of travoprost preserved with sofZia, latanoprost with $0.02 \%$ benzalkonium chloride, and preservative-free artificial tears.Cornea. 2008;27(3):339-343.

[3]. Kahook MY, Noecker R. Quantitative analysis of conjunctival goblet cells after chronic application of topical drops. Adv Ther. 2008;25(8): 743-751.

[4]. Horsley MB, Kahook MY. Effects of prostaglandin analog therapy on the ocular surface of glaucoma patients. Clin Ophthalmol. 2009;3:291-295.

[5]. Lewis RA, Katz GJ, Weiss MJ et al. (2007): Travoprost $0.004 \%$ with and without benzalkonium chloride: a comparison of safety and efficacy. J Glaucoma 16: 98-103.

[6]. Henry JC, Peace JH, Stewart JA \& Stewart WC (2008): Efficacy, safety, and improved tolerability of travoprost BAK-free ophthalmic solution compared with prior prostaglandin therapy. Clin Ophthalmol 2: 613-621.

[7]. Rossi GC, Tinelli C, Pasinetti GM, et al. Dry eye syndrome-related quality of life in glaucoma patients. Eur J Ophthalmol. 2009;19(4):572-579.

[8]. $\quad$ Leung EW, Medeiros FA \& Weinreb RN (2008): Prevalence of ocular surface disease in glaucoma patients. J Glaucoma 17: 350-355.

[9]. Michael J Miyashiro,Samuel C Lo,Jeanette A Stewart,William C Stewart(2010): Efficacy, safety, and tolerability of travoprost $0.004 \%$ BAK-free versus prior treatment with latanoprost 0.005\% in Japanese patients. Clinical Ophthalmology 2010:4 1355-1359 